\title{
The availability of probiotics and donor human milk is associated with improved survival in very preterm infants
}

\author{
Janet Sharpe ${ }^{1} \cdot$ Mandy Way $^{2} \cdot$ Pieter J. Koorts $^{1} \cdot$ Mark W. Davies $^{1,3}$
}

Received: 17 January 2018 / Accepted: 29 May 2018 / Published online: 27 June 2018

(c) Children's Hospital, Zhejiang University School of Medicine 2018

\begin{abstract}
Background To determine whether the introduction of pasteurized donor human milk and probiotics for infants born $<32$ weeks gestational age or $<1500 \mathrm{~g}$ birthweight is associated with a reduction in mortality and the incidence of necrotising enterocolitis (NEC) and sepsis.

Methods We performed a retrospective analysis of two cohorts: before and after the introduction of probiotics and pasteurised donor human milk. Univariate analysis of primary and secondary outcomes was performed; variables impacting outcomes were assessed using multivariate logistic regression.

Results There were 1791 infants: 1334 in the pre-donor milk/probiotic cohort and 457 in the post-donor milk/probiotic cohort. On univariate analysis, mortality ( 7.6 vs. $2.4 \%, P<0.001)$ and incidence of sepsis $(6.2$ vs. $3.5 \%, P=0.028)$ were statistically significantly lower in the post-donor milk/probiotic group. NEC ( 2.8 vs. $1.5 \%, P=0.14)$ and non-NEC associated gastrointestinal perforation ( 1.6 vs. $0.4 \%, P=0.052)$ were lower in the post-donor milk/probiotics cohort, but these were not statistically significant. The difference in mortality remained statistically significant on multivariate analysis in the post-donor milk/probiotic cohort compared to those in the pre-donor milk/probiotic cohort (odds ratio $0.31,95 \%$ confidence interval 0.16-0.61). The decrease in the incidence of NEC was consistent with previous observational studies but the difference was not statistically significant.

Conclusion The availability of probiotics and pasteurised donor human milk is associated with a reduction in mortality in very preterm infants.
\end{abstract}

Keywords Enterocolitis, necrotizing $\cdot$ Infant, newborn $\cdot$ Milk, human $\cdot$ Milk banks $\cdot$ Probiotics

\section{Introduction}

Despite decreased mortality and morbidity in very preterm and very low birth weight infants, some of them still die; and necrotising enterocolitis (NEC) and infection continue to be ongoing causes of death and chronic illness in this vulnerable group [1].

Mark W. Davies

mark.davies@health.qld.gov.au

1 Grantley Stable Neonatal Unit, Department of Neonatology, Royal Brisbane and Women's Hospital, Butterfield St, Herston, Brisbane, QLD 4029, Australia

2 Queensland Institute of Medical Research and Royal Brisbane and Women's Hospital Statistical Unit, Brisbane, QLD, Australia

3 Department of Pediatrics and Child Health, The University of Queensland, Brisbane, QLD, Australia
The use of probiotic supplementation for very low birth weight infants has been shown to reduce the rate of NEC and all-cause mortality [2-6]; possibly due to improved intestinal maturation, down-regulation of intestinal inflammation and direct competition for host binding-sites and nutrients $[7,8]$. Some trials have also shown a decreased time to full enteral feeds in infants given probiotics $[4,8]$. Importantly, no increased risk of sepsis due to probiotic bacterial species has been documented in any trials [2-6]. One retrospective cohort study did show an increase in NEC [9].

It is well established that breast milk from the infant's own mother is protective against both NEC and death in preterm infants, due to the presence of active enzymes which promote gastrointestinal mucosal maturation $[10,11]$, and immunomodulating and anti-microbial factors [12]. Provision of pasteurized human donor milk to infants whose mothers are unable to supply sufficient breastmilk reduces the risk of NEC compared with feeding with formula [13-15]. 
Multiple randomized controlled trials and cohort analyses have been performed investigating the effects of probiotics and pasteurized donor human milk provision individually in preterm infants [3-7, 9, 12-22]; however, currently no data exists on the benefits of combined probiotic use and donor breast milk supplementation. Subgroup analysis in the systematic review by Aceti et al. did find that probiotics only prevented late-onset sepsis in infants fed human milk [20].

Our nursery introduced prophylactic probiotics, in the form of Lactobacillus acidophilus and Bifidobacterium bifidum (Infloran ${ }^{\circledR}$ capsules-L. acidophilus LA37 and B. bifidum BB07-Laboratorio Farmaceutico, Mede, Italy), for all infants less than 32 weeks gestation, and pasteurized donor human milk for infants less than 34 weeks gestation or less than $1500 \mathrm{~g}$ birthweight, in September and October 2012 , respectively. We hypothesized that the joint introduction of pasteurized donor human milk and probiotics would result in a reduction in overall mortality and the incidence of NEC and sepsis, as well as improved short term outcomes including reduced time to establishment of full enteral feeds and central venous catheter use.

\section{Methods}

We performed a retrospective analysis of two cohorts: before and after the introduction of probiotics and pasteurised donor human milk supplementation. The infants studied were admitted to the Grantley Stable Neonatal Unit with a gestational age of $<32$ weeks or a birthweight of $<1500 \mathrm{~g}$. The pre-donor milk/probiotic cohort included babies born in the 5 years from 1 July 2007 to 30 June 2012. The post-donor milk/probiotic cohort included babies born in the 2 years from 1 January 2013 to 31 December 2014. Probiotics were given to infants $<32$ weeks, after informed parental consent, for a total of 42 days. Probiotics were withheld if enteral feeds were withheld, or ceased on treating clinician discretion, or if the infant was back-transferred out of the neonatal unit. Pasteurized donor human milk was provided to infants $<34$ weeks gestational age or $<1500 \mathrm{~g}$ birthweight after informed parental consent, and only given if mother's own milk was not available. Infants with major congenital abnormalities, and infants who were transferred to GSNU after receiving the majority of their neonatal intensive care at another unit were excluded.

Infants meeting the inclusion criteria were identified from the neonatal unit database. All infants were included on an intention to treat basis, regardless of whether donor milk and/or probiotics were actually received. Data for all variables listed in Table 1 were recorded (including major risk factors for, and associations with, mortality, infection and NEC). Sepsis was defined as a positive blood culture at the time of a clinical deterioration, which was treated with a course of intravenous antibiotics appropriate for that organism [23]. NEC was diagnosed in babies with at least stage 2 disease on the Modified Bell Criteria [24]. Mortality was defined as death prior to discharge home.

Primary outcomes investigated were mortality, and the incidence of NEC and sepsis. Secondary outcomes included the postnatal age at full enteral feeds (defined as $150 \mathrm{~mL} / \mathrm{kg}$ / day), duration of ventilation, total duration of respiratory support (including any mechanical ventilation, continuous positive airway pressure or high-flow nasal cannula use), duration of peripherally inserted central venous line (CVL) use, and the incidence of NEC associated gastrointestinal perforations and non-NEC associated gastrointestinal perforations.

\section{Sample size}

For univariate analysis a sample size calculation (http://clinc alc.com) using pre-intervention data for mortality in our unit (8\%) showed that 388 in the intervention group (post-donor milk/probiotics) and 1175 in the control group (pre-donor milk/probiotics) would give $80 \%$ power to detect a $50 \%$ decrease in mortality $(\alpha=0.05)$. Slightly smaller numbers (347 and 1043) would be adequate to show a $60 \%$ decrease in sepsis (pre-intervention sepsis in our unit 6\%); and much larger numbers (1082 and 3250) would be adequate to show a 50\% decrease in NEC (pre-intervention NEC in our unit $3 \%$ ). Cohorts of 5 years in the pre-donor milk/probiotics group and 2 years in the post-donor milk/probiotics group would give approximately adequate numbers (for mortality and sepsis, but not NEC) with a pre/post-ratio of about 3:1.

\section{Statistical analysis}

The demographics are summarised by counts and percentages for categorical variables and means and standard deviations for normally distributed continuous variables; medians and interquartile range were used otherwise. Associations between categorical variables were assessed using Pearson's Chi-squared test of association. Differences between means were compared using Student's $t$ test. Differences between medians were compared using the Mann-Whitney test.

Variables impacting outcomes were assessed using multivariate logistic regression, allowing estimation of odds ratios (OR) and 95\% confidence intervals (CI). For the logistic regression: Apgar score at 5 minutes was categorised into two groups, Apgar scores $>7$ and $\leq 7$; duration of ventilation was categorised into three groups ( $0,1-168$, and $>168$ hours); duration of CVL use was also categorised into three groups $(0,1-14$, and $>14$ days $)$; umbilical artery catheter inserted or not; postnatal age at reaching full enteral feeds was categorised into two groups (4-14 and $>14$ days). For the outcomes of sepsis and NEC, babies that died $<7$ days after birth were excluded from the analyses $(n=50)$. Pre- or post-donor milk/ probiotic cohort, gestation, sex, CVL duration (as classed 
Table 1 Data collected for infants per cohort (including major risk factors for, and associated with, mortality, infection and necrotising enterocolitis) and outcomes

\begin{tabular}{|c|c|c|c|}
\hline All infants & $\begin{array}{l}\text { Pre-donor milk/probiot- } \\
\text { ics }(n=1334)\end{array}$ & $\begin{array}{l}\text { Post-donor milk/probiot- } \\
\text { ics }(n=457)\end{array}$ & $P$ value \\
\hline Birthweight (g), mean (SD) & $1236.8(387.2)$ & $1255.2(364.9)$ & 0.374 \\
\hline Gestational age (wk), mean (SD) & $29.1(2.4)$ & $29.4(2.3)$ & 0.020 \\
\hline Apgar $5 \min \leq 7, n(\%)$ & $491(36.9)$ & $156(34.7)$ & 0.391 \\
\hline Died before discharge, $n(\%)$ & $101(7.6)$ & $11(2.4)$ & $<0.001$ \\
\hline Sepsis, $n(\%)$ & $83(6.2)$ & $16(3.5)$ & 0.028 \\
\hline Necrotising enterocolitis (NEC), $n(\%)$ & $37(2.8)$ & $7(1.5)$ & 0.139 \\
\hline NEC associated gastrointestinal perforation, $n(\%)$ & $19(1.4)$ & $6(1.3)$ & 0.861 \\
\hline Non-NEC associated gastrointestinal perforation, $n(\%)$ & $22(1.6)$ & $2(0.4)$ & 0.052 \\
\hline Patent ductus arteriosus, $n(\%)$ & $350(26.2)$ & $132(28.9)$ & 0.271 \\
\hline Umbilical arterial catheter, $n(\%)$ & $802(60.1)$ & $234(52.2)$ & 0.003 \\
\hline Infants who survived to discharge & $\begin{array}{l}\text { Pre-donor milk/probiot- } \\
\text { ics }(n=1233)\end{array}$ & $\begin{array}{l}\text { Post-donor milk/probiot- } \\
\text { ics }(n=446)\end{array}$ & $P$ value \\
\hline Age at full enteral feeds (d), median (IQR) & $13(11)$ & $11(9)$ & $<0.001$ \\
\hline \multicolumn{4}{|l|}{ Age at full enteral feeds by category (d), $n(\%)$} \\
\hline$\leq 14$ & $600(59.2)$ & $328(74.4)$ & \multirow[t]{2}{*}{$<0.001$} \\
\hline$>14$ & $413(40.8)$ & $113(25.6)$ & \\
\hline Duration of CVL days (d), median (IQR) & $9(15)$ & $7(12)$ & $<0.001$ \\
\hline \multicolumn{4}{|l|}{ Duration of CVL by category (d), $n(\%)$} \\
\hline 0 & $502(39.2)$ & $191(42.0)$ & \multirow[t]{3}{*}{$<0.001$} \\
\hline $1-14$ & $454(35.4)$ & $201(44.2)$ & \\
\hline$>14$ & $326(24.4)$ & $63(13.8)$ & \\
\hline Duration of ventilation, median (IQR) & $23(95)$ & $13(50)$ & $<0.001$ \\
\hline \multicolumn{4}{|l|}{ Duration of ventilation by category (h), $n(\%)$} \\
\hline 0 & $470(36.5)$ & 199 (44. 6) & \multirow[t]{3}{*}{0.002} \\
\hline $1-168$ & $541(42.1)$ & $180(40.4)$ & \\
\hline$>168$ & $275(21.4)$ & $67(15)$ & \\
\hline Total duration of respiratory support (h), median (IQR) & $158(867)$ & $90(578)$ & 0.001 \\
\hline
\end{tabular}

$C V L$ central venous line, $S D$ standard deviation, $I Q R$ interquartile range

above), ventilation duration (as classed above), no vs. presence of any umbilical artery catheter, and age at full enteral feeds (as defined above) were assessed for impact.

To investigate, post hoc, for improvements over time, the pre-donor milk/probiotic cohort was divided into two equal groups of 2.5 years and logistic regression predicting mortality was repeated comparing each cohort with the post-donor milk/probiotic cohort.

Ethics approval was granted by the Royal Brisbane and Women's Hospital Human Research Ethics Committee.

\section{Results}

There were 1791 infants included in the analysis: 1334 in the pre-donor milk/probiotic cohort and 457 post-donor milk/probiotic cohort. Demographics of the two groups are displayed in Table 1.
The comparison between groups on univariate analysis are shown in Table 1. Mortality and the incidence of sepsis, NEC, and non-NEC associated gastrointestinal perforation were lower in the post-donor milk/probiotics cohort, but the differences in NEC and non-NEC associated gastrointestinal perforation were not statistically significant. The decrease in the incidence of NEC was consistent with previous observational studies [21]; but the difference was not statistically significant due to the sample size.

There were also differences between the cohorts for postnatal age at reaching full enteral feeds, the duration of ventilation, total duration of ventilator support and the duration of peripherally inserted CVL use and whether a UAC was inserted or not.

In the 457 babies in the post-donor milk/probiotic cohort, 309 (68\%) received donor human milk, $370(81 \%)$ received probiotics, $264(58 \%)$ received both and $42(9 \%)$ received neither. 
Table 2 Logistic regression predicting neonatal mortality from sex, pre- or post-donor milk/probiotic cohort and gestational age

\begin{tabular}{lllll}
\hline Variables & $P$ value & OR & \multicolumn{2}{l}{$95 \%$ CI for OR } \\
\cline { 4 - 5 } & & & Lower & Upper \\
\hline Gender & & & & \\
Female & Reference & 1.000 & & \\
Male & 0.056 & $1.535^{\mathrm{a}}$ & 0.989 & 2.381 \\
Cohort & & & & \\
Pre-donor milk/probiotic & Reference & 1.000 & & \\
Post-donor milk/probiotic & 0.001 & 0.313 & 0.160 & 0.612 \\
Gestational age (wk) & $<0.001$ & $0.501^{\mathrm{b}}$ & 0.449 & 0.560 \\
\hline
\end{tabular}

$O R$ odds ratio, $C I$ confidence interval. ${ }^{\text {a }}$ The odds of death were 1.5 times higher for male compared to female infants (not statistically significant). ${ }^{\mathrm{b}}$ The odds of death were halved for each additional week of gestation

The difference in mortality remained statistically significant when adjusting for gestational age and gender on logistic regression (Table 2). The odds of mortality were reduced by $69 \%$ for infants after introduction of donor milk and probiotics in the post-donor milk/probiotic cohort compared to those in the pre-donor milk/probiotic cohort (OR $0.31,95 \% \mathrm{CI}$ $0.16-0.61)$. There was no statistically significant difference in mortality rates for infants born in the first 2.5 years of the predonor milk/probiotics cohort, compared with infants born in the second half of this period (OR 1.06, 95\% CI 0.67-1.68).

After logistic regression of infants who survived at least 7 days the difference in incidence of sepsis was no longer statistically significant (OR $0.79,95 \%$ CI $0.44-1.42$ ) when adjusting for associated variables (Table 3 ).

After logistic regression of infants who survived at least 7 days the difference in incidence of NEC was still not statistically significant (OR $0.92,95 \%$ CI $0.32-2.62$ ) when adjusting for associated variables (Table 4 ).

\section{Discussion}

Following the combined introduction of probiotics and pasteurized donor human milk we have seen a significant decrease in mortality in very preterm and very low birthweight infants. We have also seen these babies achieve full enteral feeds earlier, with reduced duration of CVL use. They spent less time on mechanical ventilation and respiratory support. There may also be clinically important reductions in the incidence of sepsis and NEC but the differences seen were not statistically significant. The reduced mortality may be explained by numerous properties of human breast milk and probiotics. Donor human milk has natural antioxidants and immunomodulating factors which persist despite the pasteurization process $[12,25,26]$. These may provide a protective effect, compared to preterm formula, against
Table 3 Logistic regression predicting sepsis from gestational age, CVL, ventilation and cohort

\begin{tabular}{lllll}
\hline Variables & $P$ value & OR & \multicolumn{2}{c}{$95 \%$ CI for OR } \\
\cline { 4 - 5 } & & & Lower & Upper \\
\hline Gestational age (wk) & $<0.001$ & $0.766^{\mathrm{a}}$ & 0.669 & 0.877 \\
CVL (d) & 0.003 & & & \\
$>14$ & Reference & 1.000 & & \\
$1-14$ & & $0.430^{\mathrm{b}}$ & 0.259 & 0.712 \\
0 & & $0.500^{\mathrm{b}}$ & 0.222 & 1.128 \\
Ventilation (h) & 0.002 & & & \\
0 & Reference & 1.000 & & \\
$1-168$ & & $3.548^{\mathrm{c}}$ & 1.362 & 9.245 \\
$>168$ & & $6.762^{\mathrm{c}}$ & 2.331 & 19.615 \\
Cohort & & & & \\
Pre-donor milk/probiotic & Reference & 1.000 & & \\
Post-donor milk/probiotic & & 0.793 & 0.443 & 1.419 \\
\hline
\end{tabular}

$O R$ odds ratio, $C I$ confidence interval, $C V L$ central venous line. ${ }^{\text {a The }}$ odds of sepsis were reduced by $23 \%$ for each additional week of gestation. ${ }^{\mathrm{b}}$ The odds of sepsis are reduced by $57 \%$ for infants who had a CVL $\leq 14 \mathrm{~d}$, compared to infants who had a CVL for $>14 \mathrm{~d}$. ${ }^{\mathrm{c}}$ The odds of sepsis were increased for increasing duration of ventilation compared with no ventilation: babies ventilated for $\leq 168$ and $>168 \mathrm{~h}$ were 3.5 and 6.8 times more likely, respectively, to develop sepsis

Table 4 Logistic regression predicting necrotising enterocolitis from gestational, CVL, age at full enteral feeds, cohort and ventilation

\begin{tabular}{lllll}
\hline Variables & $P$ value & OR & \multicolumn{2}{c}{$95 \%$ CI for OR } \\
\cline { 4 - 5 } & & & Lower & Upper \\
\hline Gestational age (wk) & 0.036 & $0.780^{\mathrm{a}}$ & 0.618 & 0.983 \\
CVL (d) & $<0.001$ & & & \\
$>14$ & Reference & 1.000 & & \\
$1-14$ & & $0.044^{\mathrm{b}}$ & 0.012 & 0.170 \\
0 & & $0.034^{\mathrm{b}}$ & 0.003 & 0.339 \\
Age at full enteral feeds (d) & 0.003 & & & \\
$\leq 14$ & Reference & 1.000 & & \\
$>14$ & & $0.246^{\mathrm{c}}$ & 0.097 & 0.624 \\
Cohort & 0.875 & & & \\
Pre-donor milk/probiotic & Reference & 1.000 & & \\
Post-donor milk/probiotic & & 0.919 & 0.322 & 2.624 \\
Ventilation (h) & & & & \\
0 & 0.543 & & & \\
1-168 & Reference & 1.000 & & \\
$>168$ & & 0.768 & 0.177 & 3.336 \\
\hline
\end{tabular}

$O R$ odds ratio, $C I$ confidence interval, $C V L$ central venous line, $N E C$ necrotising enterocolitis. ${ }^{\text {TT }}$ The odds of NEC are reduced by $22 \%$ for each additional week of gestation. ${ }^{\mathrm{b}}$ The odds of NEC are reduced by $97 \%$ in infants with no CVL compared to infants who had a CVL for $>14 \mathrm{~d}$ duration. ${ }^{\mathrm{c}}$ The odds of NEC are reduced by $75 \%$ for those infants who took $>14 \mathrm{~d}$ to reach full enteral feeds compared with infants who reached full enteral feeds at $<14 \mathrm{~d}$ 
conditions caused (in part at least) by oxygen free radical processes [17, 27]: for example neonatal chronic lung disease (arguably reflected by the reduction in duration of ventilation) that could result in death prior to discharge. Although some bioactive milk molecules are inactivated by pasteurization, some immunomodulating biological compounds found in breastmilk, such as oligosaccharides, immunoglobulins and long chain fatty acids, persist after heating [12, 23, 25], and these as well as the competitive bacterial colonization afforded by probiotics [7], may also provide protection against bacterial and viral infection. It is important to note that as both interventions were introduced at the same time that the effects of each intervention cannot be separated.

The combination of donor human milk and probiotics failed to demonstrate a statistically significant reduction in the incidence of NEC and sepsis. However, we speculate that they may have decreased the severity in these conditions when they did occur, allowing more infants to survive. Although there were still episodes of NEC, the provision of probiotics and donor milk may have placed the infant in an improved condition to limit the invasiveness, extent and ultimately, the severity of the enterocolitis. The interventions will have allowed a comparatively more mature and functional gastrointestinal tract $[12,13]$, with a higher level of competitive colonization by commensal organisms [7, 19] and modulation of the pro-inflammatory responses [7, 8], limiting the virulence and colonic extent of the pathogenic bacteria to invade, and thus a less severe infection, with fewer episodes becoming fulminant.

The near halving of the incidence of NEC, although not statistically significant, is clinically important and is consistent with previous observational studies [21]. This suggests that the availability of donor milk and probiotics is still worthwhile in neonatal units with a low baseline incidence of NEC.

Not only are more preterm infants surviving, but the infants surviving have overall shorter durations of ventilation and central venous line use in the post-donor human milk/probiotics cohort. Shorter durations of ventilation and central venous line use are associated with improved outcomes [28-31]. This suggests that infants are surviving in improved condition requiring reduced amount of prolonged critical care interventions. This will likely convert into less comorbidities and reduced length of hospital stay. These were not assessed as part of our study; however, further research in these areas would be warranted.

Time to development of full enteral feeds, along with reduced duration of central venous line use, are markers of feed tolerance. Our study demonstrated that the use of probiotics and donor human milk improved feed tolerance in preterm infants. This is likely through mechanisms including improved maturation of the gut lining due to enzymes and oligosaccharides found in donor breast milk [12, 25], improved gastric motility, and protection of the gut from pathogenic bacterial colonization with the use of probiotics
$[7,8,18]$. Improved feed tolerance is a well known benefit of breast milk [12, 13, 15] and probiotics [4] in preterm infants, and our study reflected these findings.

The differences seen could be due to factors other than the introduction of probiotics and pasteurized donor human milk. There may be unknown differences between the two cohorts. Nevertheless, the results are consistent with previous metaanalyses [2-6, 8]. Changes in management strategies (e.g., ventilation techniques) and technology between the two study periods have occurred, however, these have been minor. Our criteria for extubation were similar between cohorts, and protocols for enteral feed advancement and central venous line placement or removal were no different. Demographic data showed birthweight was comparable between the cohorts; however, infants in the post-donor human milk/probiotics cohort were slightly more mature, however, this is unlikely to have had clinical significance. The lack of a statistically significant difference in mortality rates when dividing the pre-donor human milk cohort into two epochs further demonstrates little improvement with time, until the introduction of donor human milk and probiotics.

There are no other retrospective cohort studies published comparing the joint introduction of these two managements, however, there was a recent meta-analysis by Olsen et al., which analysed 12 cohort studies investigating outcomes of the introduction of probiotics [21]. This showed a significantly reduced incidence of NEC with a RR of 0.55, and significantly reduced incidence of mortality with a risk reduction of 0.72 . Sepsis incidence was not significantly reduced. This meta-analysis shows similar results to ours, when comparing the significant mortality reduction and trend towards NEC reduction. This meta-analysis included studies with infants $<35$ weeks and $<2500 \mathrm{~g}$, and the studies used multiple different formulations of probiotics so gives a degree of similarity, however, not a direct comparison.

Similar to that analysis, our results also reflect meta-analyses of RCTs investigating both pasteurised donor human milk compared with preterm formula [13-15], and probiotics [2-6, 8], which show reduced mortality and NEC, demonstrating that clinical results from RCTs and observational analyses around the world can be reproduced in a tertiary level neonatal intensive care unit in Australia.

Given that this study has shown that the availability of probiotics and pasteurised donor human milk is associated with a reduction in mortality in very preterm infants, the continued use of probiotics and pasteurized donor human milk is supported in our neonatal intensive care unit.

In conclusion, our cohort study demonstrates significantly reduced mortality, with improved feed tolerance and reduced duration of ventilation and central venous line use, following the introduction of probiotics and pasteurized donor human milk in very preterm and very low birth weight infants. The ongoing use of probiotics and pasteurised donor human milk is supported. 
Acknowledgements Many thanks to Dr. David Cartwright who extracted data from the neonatal database, and Dr. Peter O'Rourke for assistance with study design.

Author contributions All authors contributed to design of the study. MW did the statistical analysis and all authors interpreted the findings. JS wrote the draft of the manuscript and the other authors revised it.

Funding This research project did not receive any funding.

\section{Compliance with ethical standards}

Ethical approval Ethics approval was granted by the Royal Brisbane and Women's Hospital Human Research Ethics Committee.

Conflict of interest No financial or nonfinancial benefits have been received or will be received from any party related directly or indirectly to the subject of this article.

\section{References}

1. Lin PW, Stoll BJ. Necrotising enterocolitis. Lancet. 2006;368:1271-83.

2. Guthmann F, Kluthe C, Buhrer C. Probiotics for prevention of necrotising enterocolitis: an updated meta-analysis. Klin Padiatr. 2010;222:284-90.

3. Jacobs SE, Tobin JM, Opie GF, Donath S, Tabrizi SN, Pirotta M, et al. Probiotic effects on late-onset sepsis in very preterm infants: a randomized controlled trial. Pediatrics. 2013;132:1055-62.

4. Deshpande G, Rao S, Patole S, Bulsara M. Updated meta-analysis of probiotics for preventing necrotizing enterocolitis in preterm neonates. Pediatrics. 2010;125:921-30.

5. Wang Q, Dong J, Zhu Y. Probiotic supplement reduces risk of necrotizing enterocolitis and mortality in preterm very-low-birthweight infants: an updated meta-analysis of 20 randomised, controlled trials. J Pediatr Surg. 2012;47:241-8.

6. AlFaleh K, Anabrees J, Bassler D. Probiotics reduce the risk of necrotizing enterocolitis in preterm infants: a meta-analysis. Neonatology. 2010;97:93-9.

7. Claud EC, Walker WA. Bacterial colonization, probiotics and necrotizing enterocolitis. J Clin Gastroenterol. 2008;42(Suppl 2):S46-52.

8. AlFaleh K, Anabrees J. Efficacy and safety of probiotics in preterm infants. J Neonatal Perinatal Med. 2013;6:1-9.

9. Kane AF, Bhatia AD, Denning PW, Shane AL, Patel RM. Routine supplementation of Lactobacillus rhamnosus GG and risk of necrotizing enterocolitis in very low birth weight infants. J Pediatr. 2018;195:73-9.e2.

10. Challacombe DN, Wheeler EE. Trophic action of epidermal growth factor on human duodenal mucosa cultured in vitro. Gut. 1991;32:991-3.

11. $\mathrm{Ma} \mathrm{L}, \mathrm{Xu}$ RJ. Oral insulin-like growth factor-I stimulates intestinal enzyme maturation in newborn rats. Life Sci. 1997;61:51-8.

12. Edwards TM, Spatz DL. Making the case for using donor human milk in vulnerable infants. Adv Neonatal Care. 2012;12:273-8.

13. Boyd CA, Quigley MA, Brocklehurst P. Donor breast milk versus infant formula for preterm infants: systematic review and metaanalysis. Arch Dis Child Fetal Neonatal Ed. 2007;92:F169-75.
14. McGuire W, Anthony MV. Donor human milk versus formula for preventing necrotizing enterocolitis in preterm infants: systematic review. Arch Dis Child Fetal Neonatal Ed. 2003;88:F11-4.

15. Quigley M, McGuire W. Formula versus donor breast milk for feeding preterm of low birth weight infants. Cochrane Database Syst Rev. 2014;CD002971.

16. Schanler RJ, Lau C, Hurst NM, Smith EO. Randomized trial of donor human milk versus preterm formula as substitutes for mothers' own milk in the feeding of extremely premature infants. Pediatrics. 2005;116:400-6.

17. Janvier A, Malo J, Barrington KJ. Cohort study of probiotics in a North American neonatal intensive care unit. J Pediatr. 2014;164:980-5.

18. Fernández-Carrocera LA, Solis-Herrera A, Cabanillas-Ayón M, Gallardo-Sarmiento RB, García-Pérez CS, Montaño-Rodríguez $\mathrm{R}$, et al. Double-blind, randomized clinical assay to evaluate the efficacy of probiotics in preterm newborns weighing less than $1500 \mathrm{~g}$ in the prevention of necrotizing enterocolitis. Arch Dis Child Fetal Neonatal Ed. 2013;98:F5-9.

19. Sari FN, Eras Z, Dizdar EA, Erdeve O, Oguz SS, Uras N, et al. Do oral probiotics affect growth and neurodevelopmental outcomes in very low-birth-weight preterm infants? Am J Perinatol. 2012;29:579-86.

20. Aceti A, Maggio L, Beghetti I, Gori D, Barone G, Callegari ML, et al. Probiotics prevent late-onset sepsis in human milk-fed, very low birth weight preterm infants: systematic review and metaanalysis. Nutrients. 2017;9:E904.

21. Olsen R, Greisen G, Schroder M, Brok J. Prophylactic probiotics for preterm infants: a systemic review and meta-analysis of observational studies. Neonatology. 2016;109:105-12.

22. Bonsante F, Iacobelli S, Gouyon J. Routine probiotic use in very preterm infants: retrospective comparison of two cohorts. Am J Perinatol. 2013;30:41-6.

23. Australian and New Zealand Neonatal Network. ANZNN Data Dictionary 2015. http://www.anznn.net/Portals/0/DataDictionarie s/ANZNN_2015_Data_Dictionary.pdf. Accessed 8 May 2018.

24. Bell MJ, Ternberg JL, Feigin RD, Keating JP, Marshall R, Barton $\mathrm{L}$, et al. Neonatal necrotizing enterocolitis. Therapeutic decisions based upon clinical staging. Ann Surg. 1978;187:1-7.

25. Bertino E, Giuliani F, Occhi L, Coscia A, Tonetto P, Marchino F, et al. Benefits of donor human milk for preterm infants: current evidence. Early Hum Dev. 2009;85:S9-10.

26. Unger S, Gibbins S, Zupancic J, O’Connor DL. DoMINO: donor milk for improved neurodevelopmental outcomes. BMC Pediatr. 2014;14:123.

27. ESPGHAN Committee on Nutrition, Arslanoglu S, Corpeleijn W, Moro G, Braegger C, Campoy C, et al. Donor human milk for preterm infants: current evidence and research directions. J Pediatr Gastroenterol Nutr. 2013;57:535-42.

28. Walsh MC, Morris BH, Wrage LA, Vohr BR, Poole WK, Tyson JE, et al. Extremely low birthweight neonates with protracted ventilation: mortality and 18-month neurodevelopmental outcomes. J Pediatr. 2005;146:798-804.

29. Doyle LW, Carse E, Adams AM, Ranganathan S, Opie G, Cheong JLY, et al. Ventilation in extremely preterm infants and respiratory function at 8 years. N Engl J Med. 2017;377:329-37.

30. Taylor JE, McDonald SJ, Tan K. Prevention of central venous catheter-related infection in the neonatal unit: a literature review. J Matern Fetal Neonatal Med. 2015;28:1224-30.

31. Stoll BJ, Hansen N, Fanaroff AA, Wright LL, Carlo WA, Ehrenkranz RA, et al. Late-onset sepsis in very low birth weight neonates: the experience of the NICHD Neonatal Research Network. Pediatrics. 2002;110:285-91. 